\title{
Polycystic Ovary Syndrome: A Brief Review with Recent Updates
}

\author{
Shahana Shermin ${ }^{1}$, Aysha Noor ${ }^{2}$, Samsad Jahan ${ }^{3}$
}

\begin{abstract}
Polycystic ovary syndrome (PCOS) is a chronic, complex and the most common endocrine disorder observed in women of reproductive age. This syndrome is heterogeneous by nature and is characterized by a combination of signs and symptoms of androgen excess and ovarian dysfunction. It is a significant public health issue. PCOS is associated with many comorbidities and also has a number of long-term metabolic and other consequences. The prevalence is quite high and is increasing day by day. It is a syndrome to be prevented by awakening awareness both in health workers and patients. There are many areas of controversies starting from its diagnosis, pathogenesis, consequences and treatment modalities. This review is an attempt to summarize the evolution of the diagnosis and current management guidelines and also to look into the future approaches. An extensive search was made through the Cochrane database, available systematic reviews and meta-analyses and recent international guidelines for providing an updated scientific overview of PCOS.
\end{abstract}

Keywords: Polycystic ovary syndrome; PCOS; hyperandrogenism.

Delta Med Col J. Jul 2019;7(2):84-99

\section{Introduction}

Polycystic ovary syndrome (PCOS) is a chronic, complex and the most common endocrine disorder observed in women of reproductive age and it also affects the adolescents. ${ }^{1}$ Up to $70 \%$ of affected women remain undiagnosed or have long delays before the condition is recognised. ${ }^{2}$ The prevalence is generally considered to be between $6-20 \%$, depending on the definition and the population studied.1,3 This syndrome is heterogeneous by nature and is characterized by a combination of signs and symptoms of androgen excess and ovarian dysfunction in the absence of other specific diagnoses. ${ }^{4}$ Women with PCOS often present in their adolescence or early adulthood with symptoms of oligomenorrhoea or hirsutism or infertility. ${ }^{5}$ Although it was previously considered as a disorder of adult women, recent evidence suggests that PCOS is a lifelong syndrome, manifesting since prenatal age. ${ }^{6}$

It is a significant public health issue. The health risks associated with PCOS, however, go far beyond management of these features and likely extend past the reproductive years through and beyond menopause. ${ }^{5}$ Women present with diverse features including psychological (anxiety, depression, body image and impaired quality of life) ${ }^{7}$, reproductive (irregular menstrual cycles, hirsutism, infertility and pregnancy complications $)^{8}$ and significant metabolic features (insulin resistance, metabolic syndrome, prediabetes, type 2 diabetes mellitus and

1. Senior Medical Officer, Dept. of Obstetrics \& Gynaecology, BIRDEM General Hospital, Dhaka, Bangladesh.

2. Senior Medical Officer, Dept. of Obstetrics \& Gynaecology, BIRDEM General Hospital, Dhaka, Bangladesh.

3. Professor, Dept. of Obstetrics \& Gynaecology, BIRDEM General Hospital, Dhaka, Bangladesh.

Correspondence: Dr. Shahana Shermin. e-mail: shahana.shermin@yahoo.com 
cardiovascular risk factors). ${ }^{9}$ There is also an increased rate of weight gain and prevalence of obesity in PCOS, increasing severity of the condition, causing considerable concern for those affected and mandating attention to healthy lifestyle. ${ }^{10}$ PCOS has the potential for serious consequences, including increased risk for the development of endometrial hyperplasia and neoplasia. ${ }^{11}$

\section{Diagnostic Criteria and Its Evolution}

Due to complexity of this disorder and various controversies, different diagnostic criteria have been proposed over last three decades. Polycystic ovary syndrome (PCOS) was first described by Stein and Leventhal in 1953 as a syndrome of oligomenorrhoea and polycystic ovaries that was variably accompanied by hirsutism, acne, and obesity. ${ }^{12}$ The first diagnostic attempt was made by the National Institutes of Health (NIH) in 1990 and included both hyperandrogenism (HA) and oligo or anovulation, as the two criteria to diagnose PCOS after excluding other related disorders. ${ }^{13}$ The Rotterdam criteria were published by Rotterdam ESHRE/ASRMsponsored PCOS Consensus Workshop Group in 2003 which adopted polycystic ovarian morphology (PCOM) (ultrasound finding of polycystic ovaries) to NIH criteria; out of these three criteria (HA, ovulation dysfunction, and PCOM), two are required to diagnose PCOS. ${ }^{13,14}$ Rotterdam criteria are the broadest and encompass all combinations of otherwise unexplained clinical or biochemical evidence of hyperandrogenism, evidence of oligo-anovulation, and PCOM. In 2006, Androgen Excess and PCOS Society (AE-PCOS) concluded that PCOS should be based only on two criteria, that is clinical and/or biochemical hyperandrogenism with either polycystic morphology or clinical anovulation. ${ }^{14,15}$ This criteria encompass otherwise unexplained hyperandrogenism with either oligo-anovulation or PCOM; and allows a diagnosis of PCOS in women with hyperandrogenism who lack anovulatory symptoms ("ovulatory PCOS"), which comprises about $10 \%$ of cases. ${ }^{15}$

The estimated prevalence of PCOS is reported to be $8.7 \%$ using the NIH criteria, rising to $17.8 \%$ under the Rotterdam criteria and $12 \%$ using the AE-PCOS definition. ${ }^{16}$ It must be emphasized that the diagnosis of PCOS is made after exclusion of other causes of androgen excess, such as non-classical congenital adrenal hyperplasia, hyperprolactinaemia, androgen-secreting ovarian or adrenal tumours, hypothyroidism, Cushing's syndrome and acromegaly. ${ }^{17}$

To meet the gaps between the diagnostic criteria, in 2012, NIH Consensus (NIH and ESHRE/ASRM) recommended broader Rotterdam/ESHRE/ASRM 2003 criteria with specific identification of PCOS phenotype of all PCOS. ${ }^{18}$ According to this recommendation, two out of three criteria (hyperandrogenism, ovulatory dysfunction, and PCOM) are needed to diagnose PCOS and each case has to be classified into a specific phenotype which are listed in Table I. ${ }^{19}$

PCOS frequently present during adolescence. Although there are three well known criteria to diagnose PCOS, they can be difficult to translate to adolescence. The diagnosis of PCOS in adolescents in not so straight forward, since anovulation and PCOM are not sufficient criteria for this purpose. Thus, clinical and biochemical evidence of hyperandrogenism and persistent oligomenorrhoea are required, especially if the latter persists beyond two years after menarche. No diagnostic criteria exist in peri and postmenopausal women. In these cases, a PCOS diagnosis can be presumed in the presence of a well-documented history of oligomenorrhoea and hyperandrogenism during their reproductive period. ${ }^{14}$ 
Table I. Diagnostic Criteria for PCOS ${ }^{20}$

\begin{tabular}{|c|c|}
\hline & It Diagnostic Criteria (Rotterdam) \\
\hline & rwise unexplained alternative phenotypes: \\
\hline 1. & Phenotype 1 (Classic PCOS) \\
\hline & a. Clinical and/or biochemical evidence of hyperandrogenism \\
\hline & b. Evidence of oligo-anovulation \\
\hline & c. Ultrasonographic evidence of a polycystic ovary \\
\hline 2. & Phenotype 2 (Essential NIH Criteria) \\
\hline & a. Clinical and/or biochemical evidence of hyperandrogenism \\
\hline & b. Evidence of oligo-anovulation \\
\hline 3. & Phenotype 3 (Ovulatory PCOS) \\
\hline & a. Clinical and/or biochemical evidence of hyperandrogenism \\
\hline & b. Ultrasonographic evidence of a polycystic ovary \\
\hline 4. & Phenotype 4 (Nonhyperandrogenic PCOS) \\
\hline & a. Evidence of oligo-anovulation \\
\hline & b. Ultrasonographic evidence of a polycystic ovary \\
\hline & lescent Diagnostic Criteria \\
\hline & rwise unexplained combination of: \\
\hline 1. & Abnormal uterine bleeding pattern \\
\hline & a. Abnormal for age or gynecologic age \\
\hline & b. Persistent symptoms for $1-2 \mathrm{y}$ \\
\hline 2. & Evidence of hyperandrogenism \\
\hline & $\begin{array}{l}\text { a. Persistent testosterone elevation above adult norms in a } \\
\text { reliable reference laboratory is the best evidence }\end{array}$ \\
\hline & $\begin{array}{l}\text { b. Moderate-severe hirsutism is clinical evidence of } \\
\text { hyperandrogenism }\end{array}$ \\
\hline
\end{tabular}

Careful clinical assessment including a meticulous history, thorough physical examination, and appropriate laboratory evaluation are mandatory for diagnosis. According to the best clinical practices guideline of 2015 by American Association of Clinical Endocrinologists (AACE) and the Androgen Excess and PCOS Society (AES), free testosterone levels are more sensitive than the measurement of total testosterone for establishing hyperandrogenism and should be ideally determined through equilibrium dialysis techniques. Value of measuring levels of androgens other than testosterone in patients with PCOS is relatively low. New ultrasound machines allow diagnosis of PCOM in patients having at least 25 small follicles ( 2 to $9 \mathrm{~mm}$ ) in the whole ovary and ovarian size of $10 \mathrm{~mL}$ remains the threshold between normal and increased ovary size. They have also mentioned that serum 17-hydroxyprogesterone and anti-Müllerian hormone $(\mathrm{AMH})$ may be useful to determine a diagnosis of PCOS. ${ }^{21}$
There are some inherent weaknesses in these criteria. Methods of commercial testosterone assay differ in different laboratories and many steroid assays are inaccurate. Moreover, serum testosterone concentration undergoes episodic, diurnal, and cyclic variation. So, the documentation of hyperandrogenaemia can be difficult and this is even more difficult in adolescents as mature levels are attained approximately 1 year after menarche. ${ }^{22}$ The sonographic definition of PCOM may lead to over diagnosis, particularly with antral follicle count criteria determined by the new generation high-definition imaging techniques. ${ }^{23}$ In adolescents, anovulatory criteria must be correlated with age and pubertal stage and routine use of PCOM is not appropriate as a diagnostic criterion. $^{14,20}$ Furthermore, all these criteria overlook the potential presence of the PCOS type of functional ovarian hyperandrogenism in patients who present with hirsutism, obesity, or insulin-resistance signs such as acanthosis nigricans, but who lack clinical evidence of ovarian dysfunction. ${ }^{24}$

\section{Aetiopathogenesis}

The aetiology of PCOS remains unclear and it is likely to be multifactorial. No single aetiologic factor fully accounts for the spectrum of abnormalities in the polycystic ovary syndrome. ${ }^{25}$ While insulin resistance (IR) and hyperandrogenism are the two key hormonal disturbances that underlies PCOS; obesity, genetic inheritance, lifestyle and environment also contribute. ${ }^{17}$ Polycystic ovaries, increased androgen levels owing to defect of the ovarian cells (most likely theca cells), and IR have hereditary components. Environmental factors either congenital or acquired include intrauterine factors such as androgen exposure and prenatal nutrition especially intrauterine growth restriction, whereas a major postnatal factor is acquired obesity influencing the phenotype. ${ }^{14,26}$ Epigenetic reprograming of foetal reproductive tissue following in utero exposure to androgens 
may trigger hypothalamic-pituitary-ovarian axis of foetus leading to altered folliculogenesis and cause PCOS in later life. ${ }^{19}$ The complex interactions between these contributing factors generally mimic an autosomal dominant trait with variable penetrance. Ethnic diversity also influences the syndrome's phenotypic diversity and its prevalence. There is a higher frequency of PCOS in Spanish, Native American and Mexican women. ${ }^{27}$

\section{Hyperandrogenism}

The ovarian theca cell synthesizes excessive amount of androgens in response to stimulation by increased luteinizing hormone in PCOS. When the concentration of luteinizing hormone increases relative to that of follicle-stimulating hormone, the ovaries preferentially synthesize androgen. ${ }^{25}$ In women with the polycystic ovary syndrome there is decrease in the ratio of luteinizing hormone to follicle-stimulating hormone as they tend to have an increased luteinizing hormone pulse frequency which favors transcription of the $\beta$-subunit of luteinizing hormone over the $\beta$-subunit of follicle-stimulating hormone. This may be due to an intrinsic abnormality in the GnRH pulse generator or by the relatively low levels of progesterone resulting from infrequent ovulation in these women. ${ }^{25}$ Studies suggest that ovarian theca cells in PCOS women more efficiently convert androgenic precursors to testosterone than normal theca cells. ${ }^{28}$

\section{Insulin Resistance}

Insulin Resistance (IR) or hyperinsulinaemia stimulates the theca cells of the ovary and acts synergistically with luteinizing hormone to produce excessive testosterone, which is responsible for the clinical symptoms of hyperandrogenism (acne, hirsutism, alopecia). ${ }^{29}$ Insulin also inhibits hepatic synthesis of sex hormone-binding globulin and thus increases the proportion of free testosterone while the total testosterone concentration is at the upper range of normal or only modestly elevated. ${ }^{25}$

\section{Inflammation}

Inflammation is thought to play a key role in PCOS. Direct correlations have been found between increased levels of inflammation markers (CRP, ferritin, leukocyte TNF- $\alpha$, IL-6, IL-18) and the development of PCOS. ${ }^{30}$ Newly emerging issues include a pathogenic correlation of the markers of iron overload with PCOS. Increased levels of ferritin and transferrin and a higher frequency of the HP2/HP2 genotype of the haptoglobin $\alpha$-chain have been observed, causing a reduction of anti-inflammatory cytokines and antioxidant molecules, leading to a state of chronic inflammatory response. ${ }^{31}$

\section{Genetic Factors}

Several lines of evidence suggest that the polycystic ovary syndrome is heritable. About $3 \%-35 \%$ of mothers and $25 \%$ of sisters of women with PCOS also have PCOS, and metabolic syndrome prevalence is high in their parents and siblings. ${ }^{32}$ Tan et al. ${ }^{33}$ emphasized the increased likelihood of IR associated with certain genes (such as INSIG2 and MC4R) and the particular impact of TCF7L2 SNP on the development of type 2 diabetes mellitus (T2DM) and body weight gain in patients with PCOS (a per-allele weight gain of $1.56 \mathrm{~kg} / \mathrm{m} 2$ ). Fica et al. ${ }^{34}$ identified insulin receptor auto-phosphorylation, reduced levels of phosphatidylinositol-3-kinase in muscle tissue and visceral adiposity as probable mechanisms. Current understanding of the pathogenesis of the syndrome suggests that it is a complex multigenic disorder. However in rare instances, single-gene mutations can give rise to the phenotype of the syndrome.

Various genome-wide association studies (GWAS) of PCOS have been conducted with inconclusive results. ${ }^{14}$ These GWAS involved genes implicated in biosynthesis and activity of androgens, metabolism (such as the insulin and insulin receptor genes) and inflammatory cytokines, such as tumour necrosis factor-alpha (TNF- $\alpha$ ) and interleukin-6 (IL-6) genes. ${ }^{25,35}$ Zhao et al. ${ }^{35}$ found that single-nucleotide polymorphism 
(SNP) rs13429458 is significantly associated with familial-based risk of PCOS and association among three loci was delineated.

Aetiopathogenesis of PCOS may be summarized as shown in Fig 1.

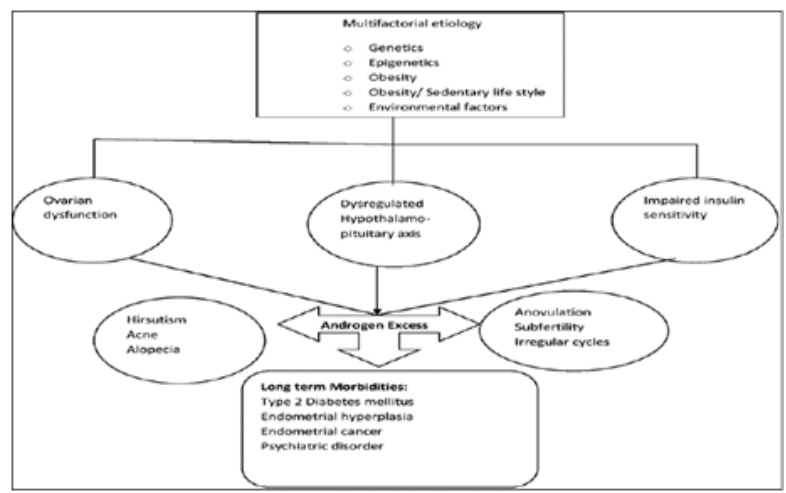

Fig 1: Aetiopathogensis of PCOS ${ }^{19}$

\section{Clinical Features}

Polycystic ovary syndrome is characterized by a combination of oligo/amenorrhoea, clinical or endocrine signs of hyperandrogenaemia and polycystic ovaries. The most common abnormalities associated with PCOS include menstrual disorders (amenorrhoea or oligomenorrhoea), often leading to infertility (in $73-75 \%$ of the cases), abdominal obesity $(30-70 \%)$ and type 2 diabetes (approximately $10 \%)^{26,34}$

Table II. Frequency of typical disorders in PCOS $^{36}$

\begin{tabular}{|l|l|}
\hline Abnormality & Frequency \\
\hline Infertility & $73-75 \%$ \\
\hline Hirsutism & $85-90 \%$ \\
\hline Metabolic syndrome: & $40 \%$ \\
\hline Abdominal obesity & $30-70 \%$ \\
\hline Diabetes type 2 & $10 \%$ \\
\hline Arterial hypertension & $20 \%$ \\
\hline Disturbed lipid metabolism & Unavailable \\
\hline
\end{tabular}

\section{Comorbidities and Long Term Health Issues}

As already mentioned, women with PCOS may present with a number of reproductive, metabolic, psychological and anthropometric complications. PCOS has metabolic implications including insulin resistance, dyslipidaemia and abnormal glucose metabolism in the form of IGT or type 2 diabetes mellitus. ${ }^{37}$ Moreover, women with PCOS also has a tendency for excess weight gain which exacerbates these symptoms. Cardiovascular risk factors such as chronic inflammation, oxidative stress and impaired fibrinolysis are increased and there is evidence that cardiovascular disease (CVD) has a higher prevalence in these women. ${ }^{38}$ Affected women are more likely to suffer from moderate to severe depression and anxiety symptoms, low self-esteem, negative body image and psychosexual function compared to healthy women. ${ }^{55}$ PCOS also negatively impacts health-related quality of life (HRQoL) and may limit a woman's ability to optimize healthy lifestyle. ${ }^{37,40}$

\section{Metabolic Consequences}

The consequences of the polycystic ovary syndrome extend beyond the reproductive problems and the syndrome is associated with significant metabolic derangements, mostly mediated by obesity and IR. The polycystic ovary syndrome might thus be viewed as a sex-specific form of the metabolic syndrome, and the term "syndrome XX" has been suggested to underscore this association. ${ }^{25}$ These consequences include increased risk of IGT and T2DM, dyslipidaemia, systemic inflammation, non-alcoholic fatty liver disease (NAFLD), hypertension and potential coagulation disorders. ${ }^{14}$

\section{Insulin Resistance and Diabetes Mellitus}

The prevalence of insulin resistance (IR) is estimated to be $30 \%$ in lean and $70 \%$ in obese women with PCOS. ${ }^{41}$ Women with PCOS have a higher risk of IR and glucose intolerance when compared with age and weight-matched healthy women. ${ }^{41,42}$ Ethnicity also seems to modify the metabolic phenotype related to IR. Hispanic 
PCOS women have a more severe phenotype and Non-Hispanic black PCOS women have the mildest phenotype. ${ }^{24}$

It has been observed that the hyperandrogenic and anovulatory phenotype is the most insulin resistant, irrespective of BMI or central adiposity. ${ }^{43}$ Observation of a large study including 1,212 women with PCOS was that phenotype1 (all three Rotterdam criteria), is associated with more severe IR and hyperandrogenaemia compared with the other three phenotypes. Phenotypes 4 (oligo or anovulation and PCOM) and 2 (hyperandrogenaemia and oligo or anovulation) showed greater IR than phenotype 3 (hyperandrogenaemia and PCOM), but did not differ from BMI-matched healthy women. ${ }^{44}$ Adrenal hyperandrogenaemia does not seem to deteriorate IR. Depending on certain risk factors, such as obesity and family history; the prevalence of IGT and T2DM in PCOS patients is 23-35\% and $4-10 \%$ respectively. In lean PCOS the respective prevalence is $10-15 \%$ and $1-2 \% .{ }^{45}$

\section{Dyslipidaemia}

Prevalence of lipid abnormalities in PCOS women is about $70 \%$ which is quite higher than healthy women. ${ }^{46}$ There is increased levels of low density lipoprotein cholesterol (LDL-C) and very-low density lipoprotein cholesterol (VLDL-C), with high serum triglyceride ( $\mathrm{Tg}$ ) and free fatty acid concentrations, as well as decreased high density lipoprotein cholesterol (HDL-C) levels, particularly HDL2-C, due to decreased apolipoprotein A-I (apoA-I). ${ }^{46,47}$ PCOS women also have higher concentrations of oxidized LDL-C, irrespective of BMI, which further increases CVD risk. ${ }^{48}$ This atherogenic profile is exacerbated by obesity and IR. ${ }^{46,47}$ Ethnic and environmental factors seem to contribute to differences in the prevalence of lipid disorders amongst PCOS women and atherogenic dyslipidaemia is more profound in obese PCOS. ${ }^{14}$

\section{Obesity}

Obesity is a key contributor to the clinical and metabolic manifestations of PCOS patients. The prevalence of obesity in women with PCOS was assessed in two systematic reviews and a greater risk of obesity was reported. ${ }^{49,50}$ Observation in a meta-analysis was that PCOS women are at two and three-fold higher risk of being overweight or obese, respectively, compared with their non-PCOS counterparts and this prevalence is also affected by ethnicity; higher in Caucasian than in Asian women. ${ }^{50}$ The body's distribution of adipose tissue is of utmost importance as abdominal obesity is an independent risk factor for CVD. The prevalence of overweight and obesity in PCOS is about $80 \%$, with a higher BMI and waist-hip ratio. ${ }^{51}$ The key role of excess weight in worsening reproductive, metabolic and psychological outcomes in PCOS has also been recognized in all the researches and analyses. ${ }^{37}$

The international guideline in PCOS recommends that BMI be assessed in all women with PCOS and that prevention of excess weight gain is vital and weight loss is recommended as the first-line treatment for overweight or obese women. ${ }^{17,52}$

\section{Metabolic Syndrome (MetS)}

Prevalence of Metabolic syndrome (MetS) is higher in PCOS patients compared with the general population (ranging from $33 \%$ to $47 \%$ in most studies, increasing to $53 \%$ in ages $30-39$ years). ${ }^{53}$ Metabolic syndrome is associated with a five-fold higher risk for T2DM and two-fold higher risk for CVD. ${ }^{54}$ There is a significant role of androgens in the pathogenesis of Mets in PCOS as the prevalence of MetS is higher in hyperandrogenic than non-hyperandrogenic anovulatory women with PCOS $(24.8 \%$ vs $0 \%){ }^{55}$

\section{Cardiovascular Disease}

In a review by Gilbert et al. ${ }^{37}$ existing systematic reviews and meta-analyses were summarized evaluating comorbidities and complications of 
PCOS. They went through a large number of reviews $(\mathrm{n}=23)$ that included 575 studies and over a million participants $(1,090,072)$. They found women with PCOS have an increased risk of surrogate markers for CVD and higher prevalence of CVD. ${ }^{37}$ Several studies indicate an increased subclinical atherosclerosis in PCOS, by various indices such as increased arterial stiffness, coronary artery calcification scores, carotid artery intima-media thickness (CIMT) and endothelial dysfunction measured by flow-mediated dilation (FMD), compared with controls, even from early reproductive ages. ${ }^{14}$ But still the association between CVD and PCOS remains controversial and findings of several prospective cohort studies are conflicting. ${ }^{56,57}$ Evidence of higher prevalence of surrogate markers of CVD predominately comes from perimenopausal women with PCOS. Almost three to four decades gap between PCOS diagnosis and CVD manifestation limits the number of large, well-phenotyped cohort studies with sufficient long-term follow-up. ${ }^{17,21,37}$ It is suggested that well designed, high quality longitudinal studies are needed, specifically focusing on the association of the clinical aspects of PCOS, such as abdominal obesity and IR with CVD risk. ${ }^{14}$

\section{Non-Alcoholic Fatty Liver Disease (NAFLD)}

When compared to healthy women, women with PCOS have an increased prevalence (27-62\%) of non-alcoholic fatty liver disease (NAFLD). ${ }^{58,59}$ Notably, an increased prevalence of PCOS has been also described in females of reproductive age with NAFLD. ${ }^{58}$ Insulin resistance seems to play the main pathogenetic role as it is associated with the development of NAFLD independently of obesity (BMI or waist circumference). ${ }^{59}$

\section{Hypertension and Vascular Dysfunction}

Women with PCOS seem to be at increased risk for development of hypertension, at least in later postreproductive life. The prevalence of hypertension is $9-25.7 \%$ in PCOS premenopausal women which is higher than the general population. ${ }^{60}$ Two main contributors are obesity and IR, and androgens play an independent pathogenetic role through activation of the rennin angiotensin system. Reduced vascular compliance and vascular endothelial dysfunction were noted in women with PCOS in a number of studies. The degree of impairment is significantly greater in obese women. ${ }^{61}$

\section{Coagulation Disorders}

PCOS has been described as a potential prothrombotic state as it is associated with disturbances in the coagulation and fibrinolysis systems like increased levels of plasminogen activator inhibitor 1 (PAI-1) and fibrinogen. ${ }^{62}$ Increased homocysteine concentrations have also been demonstrated in PCOS, independent of BMI. 63

Oral contraceptive pills (OCP) augment the risk of venous thromboembolism in women with PCOS (three to six fold higher RR) as there is an inherent prothrombotic state. The risk depends on the oestrogen dose and the generation of OCP (higher with $30 \mu \mathrm{g} / \mathrm{d}$ of ethinyl-oestradiol and third generation OCP). ${ }^{64}$ OCP may increase systemic inflammation, increase IR and alter lipid profile, by increasing TG and HDL-C and decreasing LDL-C/HDL-C ratio and may be weakly associated with increased CVD risk, although data are inconsistent and contradictory. ${ }^{64}$ Progestogen only pills or hormone releasing intrauterine devices are relatively safer. The risk is increased only when progestogen is combined with ethinyl-oestradiol and is lowest with levonogestrel. 65

\section{Infertility}

Women with PCOS may have reduced fertility due to ovulatory disorders and other endocrine abnormalitues. ${ }^{6}$ A recent study showed that infertility is 10 times more common among women with PCOS in comparison to healthy 
controls. ${ }^{66}$ The influence of individual PCOS phenotype on female fertility remains poorly comprehended. ${ }^{6}$

\section{Pregnancy Outcome}

Women with PCOS have a negative effect on pregnancy outcome as it is suggested by an increasing body of evidence. They are at increased risk of developing gestational diabetic complications. Moreover, the foetal nutrition and endocrine environment (e.g. hyperinsulinaemia) may affect foetal body weight, food intake and metabolism, with consequent long-term health in the offspring. ${ }^{8}$ Women with PCOS have an increased risk of adverse pregnancy, foetal and neonatal outcome including preeclampsia, pregnancy induced hypertension, preterm delivery, caesarean delivery, miscarriage, perinatal death, neonatal hypoglycaemia and GDM which is in line with the current evidence-based guidelines and specialty society position statements. ${ }^{37,52}$

\section{Association with Cancer}

A significant increase in risk of endometrial cancer in women with PCOS has been addressed in two systematic reviews. ${ }^{11}$ This may be the result of endometrial proliferation underlined by insulin resistance and oligomenorrhoea, prolonged endometrial exposure to unopposed oestrogen in anovulation and/or related risk factors such as obesity and T2DM. ${ }^{67}$ Breast and ovarian cancer also have been variably associated with PCOS. 68 PCOS patients frequently have associated obesity, anovulation, infertility, and receive hormonal treatment for infertility. So it is quite difficult to identify an independent risk factor for these types of cancer. ${ }^{25}$

\section{Psychological Disorders}

Women with PCOS have a lower quality of life, and an increased prevalence of depressive and anxiety symptoms which has a positive correlation with higher BMI as observed in a number of systematic reviews and meta-analyses. $37,40,69,70$ This signifies the prominent role of weight reduction in the first-line management of PCOS. Weight reduction could potentially improve mood also as reported in clinical trials. ${ }^{71,72}$ The psychological problems in PCOS may relate to coping with issues such as femininity, sexuality and fertility, overweight and the nature of living with a chronic disease. ${ }^{40,49}$ International surveys suggest that most patients of PCOS are dissatisfied with long-term counseling related to medical and psychologic issues. ${ }^{5}$ So, women with PCOS should be counseled appropriately about the increased risk of moderate and severe depressive and anxiety symptoms.

\section{Obstructive Sleep Apnea}

Recent studies indicate that the prevalence of obstructive sleep apnea in the polycystic ovary syndrome is higher than expected and cannot be explained by obesity alone. Insulin resistance appears to be a stronger predictor of sleep-disordered breathing than is age, body-mass index, or the circulating testosterone concentration. ${ }^{25,73}$

\section{Screening Recommendations}

Only to treat current symptoms is not sufficient in PCOS, but we must try to prevent any long-term morbidity. So screening recommendations are an essential part of the management of PCOS.

\section{Screening for Type 2 DM and GDM}

European Society of Endocrinology (ESE) recommends an oral glucose tolerance test (OGTT) in all obese PCOS women and in lean PCOS of $>40$ years, with a history of GDM or family history of T2DM. ${ }^{6,17}$ On the contrary, the Endocrine Society and ESHRE/ASRM recommend to perform OGTT in all adolescents and adult women with PCOS, due to their high risk in developing IGT and T2DM. Neither of the societies recommend the use of hemoglobin A1c 
(HbA1c) as a screening test. ${ }^{74}$ The screening should be done every 3-5 years ${ }^{74}$, or every second year in patients with no risk factors for type $2 \mathrm{DM}$ and annually in patients with risk factors. ${ }^{75}$ Recent international guideline in PCOS recommends an OGTT preconception or early in pregnancy at 24 to 28 week gestation. 52

\section{Screening for CVD}

Despite the controversies, current international guidelines recommend that all women with PCOS should be screened for individual CVD risk factors. ${ }^{17,21,37}$ Guidelines recommend cigarette smoking assessment, body weight and BMI measurements, blood pressure monitoring, and a complete lipid profile panel. ${ }^{74,75}$ The Australian Guideline emphasizes CVD screening recommendations, including blood pressure measurement annually if $\mathrm{BMI} \leq 25 \mathrm{~kg} / \mathrm{m}^{2}$ or at each visit if $\mathrm{BMI} \geq 25 \mathrm{~kg} / \mathrm{m}^{2}$ and lipid profile assessment every 2 years if initially normal or every year if initially abnormal..$^{75}$

\section{Screening for Psychological Wellbeing}

Women with PCOS should be screened at diagnosis and potential risk factors for psychological disturbances should be identified. ${ }^{76}$ Guidelines recommend these women should be screened for not only depression and anxiety but also for negative body image, eating disorders, and psychosexual dysfunction. ${ }^{74,75}$ If screening is positive, further assessment by health physician and referral to a specialist is recommended. A few studies indicate an increased risk of depression in adolescents also and this group warrants screening. ${ }^{76}$

Though NAFLD is also associated with increased risk of CVD, current guidelines and specialty societies have not recommended any routine screening for NAFLD in women with PCOS at this stage, as long-term health outcomes remain unclear. $^{74}$

\section{Treatment}

The management of PCOS targets the symptomatology for which patients usually present. Treatment should be offered involving a multidisciplinary approach including a family practitioner, a gynaecologist, an endocrinologist, a dermatologist, a paediatrician, a nutritionist, a psychiatrist, and a psychologist.

The treatment recommendations are mainly adopted here from two major treatment guidelines: the American Task Force and the PCOS Australian Alliance Guidelines. ${ }^{74,75}$ Other recent guidelines are also considered.

\section{Lifestyle Changes}

Lifestyle modifications are considered as a cost-effective first line treatment. Guidelines recommend exercise therapy and calorie-restricted diet as a crucial part of the management of obesity in women with PCOS. ${ }^{74,75}$ A number of clinical trials have shown that a body weight decrease of as little as 5\% regulates the menstrual cycle, improves fertility, reduces insulin and testosterone levels, decreases the degree of acne and hirsutism, and benefits psychological wellbeing. ${ }^{77-79}$

\section{Medical Treatment}

Medical treatment is added if lifestyle changes are not enough to resolve symptomatology.

\section{Oral Contraceptive Pills}

Oral contraceptive pills (OCP) are the most commonly used medications for the long-term treatment of women with PCOS and have been recommended by the Task Force and the Endocrine Society, the Australian Alliance, and the PCOS Consensus Group as first-line treatment for hyperandrogenism and menstrual cycle irregularities in women with PCOS. ${ }^{18,74,75}$

Oral contraceptive pills (OCP) decrease LH secretions, increase sex hormone binding globulins, and decrease free testosterone levels by 
suppressing the hypothalamo-pituitary-ovarian axis. This addresses hyperandrogenism-mediated symptoms; improves acne and hirsutism and corrects menstrual cycle abnormalities. ${ }^{80} \mathrm{~A}$ minimum of 6 months of OCP regimen is usually required to obtain satisfactory results against acne and hirsutism. Low-dose OCP that contain anti-androgenic or neutral progestins are the best choice. ${ }^{81}$

\section{Metformin}

Metformin is an oral anti-diabetic biguanide drug. Even though studies show contradictory results regarding metformin effect, it is suggested as first-line treatment for cutaneous manifestations and pregnancy complications in women with PCOS. It is also used as a combination with clomiphene citrate to improve fertility outcomes in clomiphene citrate resistant patients. ${ }^{74,75}$

\section{Antiandrogens}

Antiandrogens mainly act either by competitive inhibition of androgen-binding receptors or inhibit 5-alpha reductase enzyme which decreases androgen production. OCPs should be added with all antiandrogens in sexually active women as there is risk of feminization of male foetus if pregnancy occurs. ${ }^{19}$

\section{Spironolactone}

It is the most effective antiandrogen which has shown demonstrable effect on hirsutism even over and above OCPs. It has been found to be effective for acne and alopecia. It is generally well tolerated and should be given in combination with OCP to avoid menstrual irregularity. ${ }^{19}$ But current guidelines do not provide any specific recommendations for the use of spironolactone in the management of PCOS.

\section{Cyproterone Acetate}

It is a progestational antiandrogen. It can be used alone in dose of $50-100 \mathrm{mg}$ daily or in combination with ethinyl-oestradiol It is generally well tolerated; however, hepatotoxicity is a rare side effect. It is usually recommended for hirsutism and alopecia. ${ }^{19}$

\section{Flutamide}

Flutamide is a nonsteroidal antiandrogen. Flutamide with metformin is found to be more effective than OCP alone in improving PCOS symptoms. But hepatotoxicity is a serious side effect with its use. ${ }^{19}$

\section{Finasteride}

Finasteride is a potent antiandrogen and is used in combination with OCPs with better results than OCPs alone. Where oestrogens are contraindicated, combination of finasteride with spironolactone has also been tried and found effective. ${ }^{19}$

\section{Cosmetic/Local Therapy}

Options available are medical therapy or physical method of removing hairs by threading, waxing, plucking, bleaching, or shaving. The permanent hair-reduction techniques, such as electrolysis, laser thermolysis and photoepilation, are also there in which destruction of hair follicle is done with energy source. ${ }^{19}$

\section{Infertility Treatment}

Both the American Task Force and the PCOS Australian Alliance Guidelines recommend clomiphene citrate as first line treatment of anovulatory infertility. ${ }^{36,74,75}$ The American College of Obstetricians and Gynecologists (ACOG) has recently issued clinical management guidelines that updated the use of letrozole for ovulation induction in women with PCOS. They have advocated letrozole as first-line therapy for ovulation induction because of the increased live birth rate compared with clomiphene citrate. ${ }^{82}$ If clomiphene citrate or letrozole use fails to result in pregnancy, the recommended second-line 
intervention is either exogenous gonadotropins, in vitro fertilization or laparoscopic ovarian surgery. 82,83 Laparoscopic techniques that can successfully trigger ovulation include ovarian biopsy and electrocautery, laparoscopic ovarian drilling, transvaginal hydrolaparoscopy, ultrasound guided transvaginal ovarian needle drilling or laparoscopic ovarian multi-needle intervention. ${ }^{17,25,36}$

\section{Treatment in Adolescents}

Till date no placebo-controlled randomized controlled trials for the treatment of PCOS in adolescents have been conducted. Recommendations suggest individualizing treatment of adolescents with PCOS. ${ }^{74}$ Lifestyle modification and weight reduction are considered as part of the first-line treatment, especially in obese adolescents. The mainstay of therapy for adolescents with PCOS is OCPs. However the best OCPs and their appropriate duration of use in adolescents are not well defined. Metformin is also widely used, yet, the necessary treatment period is still indefinite. ${ }^{6}$ Early lifestyle modifications and metformin therapy have been associated with promising preventative results. ${ }^{74}$

\section{New Therapeutic Options}

In a study by Cakir et al. ${ }^{84}$, women with acne received intramuscular injections of 0.5-1 $\mathrm{mg} / \mathrm{kg} / \mathrm{dL}$ isotretinoin, and the therapy was highly effective. It may be the used as a first-line treatment for PCOS patients with acne, second only to oral contraceptive therapy. Isotretinoin may also improve the patients' reduced AMH levels. However, Isotretinoin therapy fails to produce the desired clinical effect in the patients with severe acne. ${ }^{85}$ It is expensive and contributes to an increase in body weight and triglyceride levels and not yet widely recommended in PCOS. ${ }^{36}$

A newer polytherapy was proposed recently by Vinaixaet al. ${ }^{86}$ including 3-month flutamide- metformin-pioglitazone therapy combined with ester-progestogen treatment, which also improved the lipid profile (reduced LDL, increased HDL), higher androgen levels as well as increased carotid intima media (CIM) thickness, which in turn prevents the occurrence of atherosclerosis and related complications.

Thiazolidinedione derivatives use is another alternative which enhances insulin sensitivity. However, these drugs do not reduce androgen levels, can contribute to patients'weight gain and are contraindicated for women wishing to become pregnant. ${ }^{87}$

Metformin combination therapy with new drugs glucagon-like peptide receptor agonists 1 (GLP-1) leads to more effective weight reduction, lowers insulin resistance and improves reproductive function. However, they do not have a high safety profile in women of reproductive age. ${ }^{88}$

It is suggested that vitamin D deficiency has an impact on the pathogenesis of insulin resistance in PCOS and several studies have recently been published on the effectiveness of vitamin D supplementation. ${ }^{89}$

As lipid disorders and obesity are frequently associated with PCOS, use of statins is promising. Statins have anti-inflammatory, antioxidant, antiproliferative and lipid-lowering effects. ${ }^{26}$ This was confirmed by the results of Celik and Acbay 90 , who demonstrated that a 12-week combination therapy with metformin in reducing the testosterone, DHEA-S, body weight, CRP, TG and LDL cholesterol. Supplementation with omega-3, $\alpha$-lipoic acid and $\mathrm{N}$-acetyl cysteine have antioxidant, anti-inflammatory effect, and also improves insulin sensitivity and lipid profile of women with PCOS. ${ }^{91}$

Increased activity of the sympathetic nervous system plays a role in the pathogenesis, progression and treatment of PCOS. Obesity, hyperinsulinemia, obstructive sleep apnea (OSA) and metabolic disorders in PCOS patients are enhanced by increased intraovarian production of nerve growth factor (NGF) and elevated muscle 
sympathetic nerve activity (MSNA). To combat these, the use of non-pharmacological interventions (weight reduction, continuous positive airway pressure in OSA, electro-acupuncture stimulation of baroreceptors), pharmacological treatment (insulin sensitizers) and surgical procedures (renal denervation) can bring surprising results. ${ }^{92}$

Studies have shown that fibroblast growth factors (FGFs), particularly FGF 1, 10, 19 and 21 are involved in the regulation of carbohydrate and lipid metabolism, have cardioprotective activity (FGF-21), and are also responsible for the excessive activity of the sebaceous glands. An analog of FGF-21 called LY2405319, is shown to reduce insulin resistance, improve dyslipdaemia and reduce weight. ${ }^{27}$

Some recent clinical trials showed that myo-inositol decreases glycemia, improves the serum lipid profile and reduces the secretion of LH, DHEA, testosterone and progesterone. A high concentration of myo-inositol in the follicular micro environment increases the number of oocytes. Therefore myo-inositol restores menstrual regularity, ovulation and improves fertility. ${ }^{93}$

\section{Conclusion}

Polycystic ovary syndrome, though the commonest endocrine pathology, till date research and extensive studies are being carried out as its aetiopathogenesis is still unclear, diagnostic critera are still evolving, management is complex and newer therapeutic options are being explored every day. Often key patient needs are not being met well, and there is gap of knowledge in both patients and health professionals. But what is important to remember and practice is that it is a syndrome more to prevent than to treat. All providers involved in the multidimensional care of women with PCOS should be aware of its long-term health risks to provide appropriate counseling, screening, and management options.

\section{References}

1. Azziz R, Carmina E, Chen Z, Dunaif A, Laven JS, Legro RS, et al. Polycystic Ovary Syndrome. Nature Reviews Disease Primers. 2016;2:16057.

2. Gibson-Helm M, Teede H, Dunaif A, Dokras A. Delayed Diagnosis and a Lack of Information Associated with Dissatisfaction in Women with Polycystic Ovary Syndrome. J Clin Endocrinol Metab. 2017;102:604-12.

3. Balen AH, Laven JS, Tan SL, Dewailly D. Ultrasound Assessment of the Polycystic Ovary: International Consensus Definitions. Hum Reprod Update. 2003;9:505-14.

4. Escobar-Morreale HF. Polycystic Ovary Syndrome: Definition, Aetiology, Diagnosis and Treatment. Nature Reviews Endocrinology. 2018;14:270-84.

5. Cooney LG, Dokras A. Beyond Fertility: Polycystic Ovary Syndrome and Long-Term Health. Fertil Steril. 2018;110(5):794-809.

6. El Hayek S, Bitar L, Hamdar LH, Mirza FG, Daoud G. Poly Cystic Ovarian Syndrome: An Updated Overview. Front Physiol. 2016;7:124. doi: 10.3389/fphys.2016.00124.

7. Teede H, Deeks A, Moran L. Polycystic Ovary Syndrome: A Complex Condition with Psychological, Reproductive and Metabolic Manifestations That Impacts on Health across the Lifespan. BMC Medicine. 2010;8:41.

8. Boomsma CM, Eijkemans MJC, Hughes EG, Visser GHA, Fauser BCJM, Macklon NS. A Meta-Analysis of Pregnancy Outcomes in Women with Polycystic Ovary Syndrome. Hum Reprod Update. 2006;12(6):673-83.

9. Teede HJ, Misso ML, Costello MF, Dokras A, Laven $\mathrm{J}$, Moran L, et al. on behalf of the International PCOS Network. International Evidence-Based Guideline for the Assessment and Management of Polycystic Ovary Syndrome 2018. Monash University: Melbourne, Australia; 2018.

10. Teede HJ, Joham AE, Paul E, Moran LJ, Loxton D, Jolley D, et al. Longitudinal Weight Gain in Women Identified with Polycystic Ovary Syndrome: Results of an Observational Study in Young Women. Obesity. 2013;21(8):1526-32.

11. Barry JA, Azizia MM, Hardiman PJ. Risk of Endometrial, Ovarian and Breast Cancer in Women with Polycystic Ovary Syndrome: A Systematic Review and Meta-Analysis. Hum Reprod Update. 2014;20(5):748-58. 
12. Azziz R, Adashi EY. Stein and Leventhal: 80 Years on. Am J Obstet Gynecol. 2016;214:247.e1-247.e11.

13. Lujan ME, Chizen DR, Pierson RA. Diagnostic Criteria for Polycystic Ovary Syndrome: Pitfalls and Controversies. J Obstet Gynaecol Can. 2008;30:671-79.

14. Anagnostis P, Tarlatzis BC, Kauffman RP. Polycystic Ovarian Syndrome (PCOS): Long-Term Metabolic Consequences. Metabolism Clinical and Experimental. 2018;86:33-43.

15. Azziz R, Carmina E, Dewailly D, Diamanti-Kandarakis E, Escobar-Morreale HF, Futterweit W, et al. The Androgen Excess and PCOS Society Criteria for the Polycystic Ovary Syndrome: The Complete Task Force Report. Fertil Steril. 2009;91:456-88.

16. March WA, Moore VM, Willson KJ, Phillips DI, Norman RJ, Davies MJ. The Prevalence of Polycystic Ovary Syndrome in a Community Sample Assessed under Contrasting Diagnostic Criteria. Hum Reprod. 2010;25:544-51.

17. Conway G, Dewailly D, Diamanti-Kandarakis E, Escobar-Morreale HF, Franks S, Gambineri A, et al, ESE PCOS Special Interest Group. The Polycystic Ovary Syndrome: A Position Statement from the European Society of Endocrinology. Eur J Endocrinol. 2014;171:1-29.

18. Fauser BC, Tarlatzis BC, Rebar RW, Legro RS, Balen AH, Lobo R, et al. Consensus on Women's Health Aspects of Polycystic Ovary Syndrome (PCOS): The Amsterdam ESHRE/ASRM-Sponsored 3rd PCOS Consensus Workshop Group. Fertil Steril. 2012;97:28-38.

19. Gainder S, Sharma B. Update on Management of Polycystic Ovarian Syndrome for Dermatologists. Indian Dermatol Online J. 2019;10:97-105.

20. Rosenfield RL. The Diagnosis of Polycystic Ovary Syndrome in Adolescents. Pediatrics. 2015;136:1154-65.

21. Goodman NF, Cobin RH, Futterweit W, Glueck JS, Legro RS, Carmina E; American Association of Clinical Endocrinologists (AACE); American College of Endocrinology (ACE); Androgen Excess and PCOS Society (AES). American Association of Clinical Endocrinologists, American College of Endocrinology, and Androgen Excess and PCOS Society Disease State Clinical Review: Guide to the Best Practices in the Evaluation and Treatment of Polycystic Ovary Syndrome-Part 1. Endocr Pract. 2015;21(11):1291-300
22. Rosenfield RL. Clinical Practice. Hirsutism. N Engl J Med. 2005;353:2578-88.

23. Dewailly D, Lujan ME, Carmina E, Cedars MI, Laven J, Norman RJ, et al. Definition and Significance of Polycystic Ovarian Morphology: A Task Force Report from the Androgen Excess and Polycystic Ovary Syndrome Society. Hum Reprod Update. 2014;20(3):334-52.

24. Ehrmann DA, Rosenfield RL, Barnes RB, Brigell DF, Sheikh Z. Detection of Functional Ovarian Hyperandrogenism in Women with Androgen Excess. N Engl J Med. 1992;327:157-62.

25. Ehrmann DA. Polycystic Ovary Syndrome. N Engl J Med. 2005;352:1223-36.

26. Wołczyński S, Zgliczyński W. Abnormalities of the Menstrual Cycle. In: Large Interna -Endocrinology. 2nd ed. Medical Tribune Poland: Warsaw; 2012. p. 561-67.

27. Kharitonenkov A, Adams AC. Inventing New Medicines: The FGF21 Story. Mol Metab. 2014;3:221-29.

28. Nelson VL, Legro RS, Strauss JF III, McAllister JM. Augmented Androgen Production Is a Stable Steroidogenic Phenotype of Propagated Theca Cells from Polycystic Ovaries. Mol Endocrinol. 1999; 13:946-57.

29. Traub ML. Assessing and Treating Insulin Resistance in Women with Polycystic Ovarian Syndrome. World J Diabetes. 2011;2(3):33-40.

30. Sathyapalan T, Atkin ST. Mediators of Inflammation in Polycystic Ovary Syndrome in Relation to Adiposity. Mediat Inflamm. 2010;2010:758656. doi: $10.1155 / 2010 / 758656$.

31. McClung J, Karl P. Iron Deficiency and Obesity: The Contribution of Inflammation and Diminished Iron Absorption. Nutr Rev. 2009;67:100-104.

32. Kahsar-Miller MD, Nixon C, Boots LR, Go RC, Azziz R. Prevalence of Polycystic Ovary Syndrome (PCOS) in First Degree Relatives of Patients with PCOS. Fertil Steril. 2001;75:53.

33. Tan S, Scherag A, Janssen OE, Hahn S, Lahner H, Dietz T, et al. Large Effects on Body Mass Index and Insulin Resistance Women with PCOS (FTO) Variants in Patients with Polycystic Ovary Syndrome (PCOS). BMC Med Genet. 2010;11:12. doi: 10.1186/1471-2350-11-12.

34. Fica S, Albu A, Constantin M, Dobri GA, Davila C. Insulin Resistance and Fertility in Polycystic Ovary Syndrome. J Med Life. 2008;1(4):415-22. 
35. Zhao H, Lv Y, Li L, Chen ZJ. Genetic Studies on Polycystic Ovary Syndrome. Best Pract Res Clin Obstet Gynaecol. 2016;37:56-65.

36. Bednarska S, Siejka A. The Pathogenesis and Treatment of Polycystic Ovary Syndrome: What's New? Adv Clin Exp Med. 2017;26(2):359-67.

37. Gilbert EW, Tay CT, Hiam DS, Teede HJ, Moran LJ. Comorbidities and Complications of Polycystic Ovary Syndrome: An Overview of Systematic Reviews. Clin Endocrinol (Oxf). 2018;89(6):683-99.

38. Murri $M$, Luque-Ramirez $M$, Insenser $M$, Ojeda-Ojeda M, Escobar-Morreale HF. Circulating Markers of Oxidative Stress and Polycystic Ovary Syndrome (PCOS): A Systemic Review and Meta-Analysis. Hum Reprod Update. 2013;19(3):268-88.

39. Barry JA, Kuczmierczyk AR, Hardiman PJ. Anxiety and Depression in Polycystic Ovary Syndrome: A Systematic Review and Meta-Analysis. Hum Reprod. 2011;26(9):2442-51.

40. Bazarganipour F, Taghavi S, Montazeri A, Ahmadi F, Chaman R, Khosravi A. The Impact of Polycystic Ovary Syndrome on the Health-Related Quality of Life: A Systematic Review and Meta-Analysis. Iran J Reprod Med. 2015;13(2):61-70.

41. Randeva HS, Tan BK, Weickert MO, Lois K, Nestler JE, Sattar N, et al. Cardiometabolic Aspects of the Polycystic Ovary Syndrome. Endocr Rev. 2012;33:812-41.

42. Cassar S, Misso ML, Hopkins WG, Shaw CS, Teede HJ, Stepto NK. Insulin Resistance in Polycystic Ovary Syndrome: A Systematic Review and Meta-Analysis of Euglycaemic-Hyperinsulinaemic Clamp Studies. Hum Reprod. 2016;31:2619-31.

43. Diamanti-Kandarakis E, Panidis D. Unravelling the Phenotypic Map of Polycystic Ovary Syndrome (PCOS): A Prospective Study of 634 Women with PCOS. Clin Endocrinol (Oxf). 2007;67:735-42.

44. Panidis D, Tziomalos K, Misichronis G, Papadakis E, Betsas G,Katsikis I, et al. Insulin Resistance and Endocrine Characteristics of the Different Phenotypes of Polycystic Ovary Syndrome: A Prospective Study. Hum Reprod. 2012;27:541-49.

45. Legro RS, Kunselman AR, Dodson WC, Dunaif A. Prevalence and Predictors of Risk for Type 2 Diabetes Mellitus and Impaired Glucose Tolerance in Polycystic Ovary Syndrome: A Prospective, Controlled Study in 254 Affected Women. J Clin Endocrinol Metab. 1999;84:165-69.
46. Legro RS, Kunselman AR, Dunaif A. Prevalence and Predictors of Dyslipidemia in Women with Polycystic Ovary Syndrome. Am J Med. 2001;111:607-13.

47. Wild RA, Painter PC, Coulson PB, Carruth KB, Ranney GB. Lipoprotein Lipid Concentration and Cardiovascular Risk in Women with Polycystic Ovary Syndrome. J Clin Endocrinol Metab. 1985;61:946-51.

48. Macut D, Damjanovic S, Panidis D, Spanos N, Glisic B, Petakov M, et al. Oxidised Low-Density Lipoprotein Concentration- Early Marker of an Altered Lipid Metabolism in Young Women with PCOS. Eur J Endocrinol. 2006;155:131-36.

49. Kaczmarek C, Haller DM, Yaron M. Health-Related Quality of Life in Adolescents and Young Adults with Polycystic Ovary Syndrome: A Systematic Review. 2016;29(6):551-57.

50. Lim SS, Davies MJ, Norman RJ, Moran LJ. Overweight, Obesity and Central Obesity in Women with Polycystic Ovary Syndrome: A Systemic Review and Meta-Analysis. Hum Reprod Update. 2012;18(6):618-37.

51. Sam S. Obesity and Polycystic Ovary Syndrome. Obes Manag. 2007;3:69-73.

52. Teede HJ, Misso ML, Costello MF, Dokras A, Laven $\mathrm{J}$, Moran L, et al.; International PCOS Network. Recommendations from the International Evidence-Based Guideline for the Assessment and Management of Polycystic Ovary Syndrome. Hum Reprod. 2018;33(9):1602-18.

53. Essah PA, Nestler JE. The Metabolic Syndrome in Polycystic Ovary Syndrome. J Endocrinol Invest. 2006;29:270-80.

54. Anagnostis P. Metabolic Syndrome in the Mediterranean Region: Current Status. Indian J Endocrinol Metab. 2012;16:72-80.

55. Goverde AJ, van Koert AJ, Eijkemans MJ, Knauff EA, Westerveld HE, Fauser BC, et al. Indicators for Metabolic Disturbances in Anovulatory Women with Polycystic Ovary Syndrome Diagnosed according to the Rotterdam Consensus Criteria. Hum Reprod. 2009;24:710-17.

56. Merz CN, Shaw LJ, Azziz R, Stanczyk FZ, Sopko G, Braunstein GD, et al. Cardiovascular Disease and 10-Year Mortality in Postmenopausal Women with Clinical Features of Polycystic Ovary Syndrome. J Womens Health (Larchmt). 2016;25:875-81. 
57. Wang ET, Cirillo PM, Vittinghoff E, Bibbins-Domingo K, Cohn BA, Cedars MI. Menstrual Irregularity and Cardiovascular Mortality. J Clin Endocrinol Metab. 2011;96:E114-18.

58. Brzozowska MM, Ostapowicz G, Weltman MD. An Association between Non-Alcoholic Fatty Liver Disease and Polycystic Ovarian Syndrome. J Gastroenterol Hepatol. 2009;24:243-47.

59. Macut D, Tziomalos K, Božić-Antić I, Bjekić-Macut J, KatsikisI, Papadakis E, et al. Non-Alcoholic Fatty Liver Disease Is Associated with Insulin Resistance and Lipid Accumulation Product in Women with Polycystic Ovary Syndrome. HumReprod. 2016;31:1347-53.

60. Elting MW, Korsen TJ, Bezemer PD, Schoemaker J. Prevalence of Diabetes Mellitus, Hypertension and Cardiac Complaints in a Follow-Up Study of a Dutch PCOS Population. Hum Reprod 2001;16:556-60.

61. Kelly CJG, Speirs A, Gould GW, Petrie JR, Lyall H, Connell JMC. Altered Vascular Function in Young Women with Polycystic Ovary Syndrome. J Clin Endocrinol Metab. 2002;87:742-46.

62. Mannerås-Holm L, Baghaei F, Holm G, Janson PO, Ohlsson C, Lönn M, et al. Coagulation and Fibrinolytic Disturbances in Women with Polycystic Ovary Syndrome. J Clin Endocrinol Metab. 2011;96:1068-76.

63. Schachter M, Raziel A, Friedler S, Strassburger D, Bern O, Ron-El R. Insulin Resistance in Patients with Polycystic Ovary Syndrome Is Associated with Elevated Plasma Homocysteine. Hum Reprod. 2003;18:721-27.

64. Domecq JP, Prutsky G, Mullan RJ, Sundaresh V, Wang AT, Erwin PJ, et al. Adverse Effects of the Common Treatments for Polycystic Ovary Syndrome: A Systematic Review and Meta-Analysis. J Clin Endocrinol Metab. 2013;98:4646-54.

65. Lidegaard $\varnothing$, Nielsen LH, Skovlund CW, Skjeldestad FE, Løkkegaard E. Risk of Venous Thromboembolism from Use of Oral Contraceptives Containing Different Progestogens and Oestrogen Doses: Danish Cohort Study 2001-9. BMJ. 2011;343:d6423.

66. Hart R, Doherty DA. The Potential Implications of a PCOS Diagnosis on a Woman's Long-Term Health Using Data Linkage. J Clin Endocrinol Metab. 2015.100:911-19.
67. Mu N, Zhu Y, Wang Y, Zhang H, Xue F. Insulin Resistance: A Significant Risk Factor of Endometrial Cancer. Gynecol Oncol. 2012;125(3):751-57.

68. Balen A. Polycystic Ovary Syndrome and Cancer. Hum Reprod Update. 2001;7:522-25.

69. Barry JA, Kuczmierczyk AR, Hardiman PJ. Anxiety and Depression in Polycystic Ovary Syndrome: A Systematic Review and Meta-Analysis. Hum Reprod. 2011;26(9):2442-51.

70. Veltman-Verhulst SM, Boivin J, Eijkemans MJ, Fauser BJ. Emotional Distress is a Common Risk in Women with Polycystic Ovary Syndrome: A Systematic Review and Meta-Analysis of 28 Studies. Hum Reprod Update. 2012;18(6):638-51.

71. Thomson RL, Buckley JD, Moran LJ, Noakes M, Clifton PM, Norman RJ, et al. Comparison of Aerobic Exercise Capacity and Music Strength in Overweight Women with and without Polycystic Ovary Syndrome. BJOG Int J Obstet Gynaecol. 2009;116(9):1242-50.

72. Moran LJ, Noakes M, Clifton PM, Wittert GA, Williams G, Norman RJ. Short-Term Meal Replacements Followed by Dietary Macronutrient Restriction Enhance Weight Loss in Polycystic Ovary Syndrome. Am J Clin Nutr. 2006;84(1):77-87.

73. Gopal M, Duntley S, Uhles M, Attarian H. The Role of Obesity in the Increased Prevalence of Obstructive Sleep Apnea Syndrome in Patients with Polycystic Ovary Syndrome. Sleep Med. 2002;3:401-404.

74. Legro RS, Arslanian SA, Ehrmann DA, Hoeger KM, Murad MH, Pasquali R, et al.; Endocrine Society. Diagnosis and Treatment of Polycystic Ovary Syndrome: An Endocrine Society Clinical Practice Guideline. J Clin Endocrinol Metab. 2013;98:4565-92.

75. Misso M, Boyle J, Norman R, Teede $H$. Development of Evidenced-Based Guidelines for PCOS and Implications for Community Health. Semin Reprod Med. 2014;32;230-40.

76. Dokras A, Stener-Victorin E, Yildiz BO, Li R, Ottey $\mathrm{S}$, Shah D, et al. Androgen Excess - Polycystic Ovary Syndrome Society Position Statement on Depression, Anxiety, Quality of Life, and Eating Disorders in Polycystic Ovary Syndrome. Fertil Steril. 2018;109:888-99.

77. Knowler WC, Barrett-Connor E, Fowler SE, Hamman RF, Lachin JM, Walker EA, et al. Reduction in the Incidence of Type 2 Diabetes with Lifestyle Intervention or Metformin. N Engl J Med. 2002;346:393-403. 
78. Pasquali R, Gambineri A, Pagotto U. The Impact of Obesity on Reproduction in Women with Polycystic Ovary Syndrome. BJOG. 2006;113:1148-59.

79. Norman RJ, Dewailly D, Legro RS, Hickey TE. Polycystic Ovary Syndrome. Lancet. 2007;370:685-97.

80. Costello M, Shrestha B, Eden J, Sjoblom P, Johnson N. Insulin-Sensitising Drugs Versus the Combined Oral Contraceptive Pill for Hirsutism, Acne And Risk of Diabetes, Cardiovascular Disease, and Endometrial Cancer in Polycystic Ovary Syndrome. Cochrane Database Syst Rev. 2007CD005552. doi:10.1002/14651858.CD005552.pub2.

81. Yildiz BO. Assessment, Diagnosis and Treatment of a Patient with Hirsutism. Nat Clin Pract Endocrinol Metab. 2008;4:294-300.

82. Polycystic Ovary Syndrome. ACOG Practice Bulletin No. 194. American College of Obstetricians and Gynecologists. Obstet Gynecol. 2018;131:e157-71.

83. Spritzer PM, Motta AB, Sir-Petermann T, Diamanti-Kandarakis E. Novel Strategies in the Management of Polycystic Ovary Syndrome. Minerva Endocrinol. 2015;40:195-12.

84. Cakir GA, Erdogan FG, Gurler A. Isotretinoin Treatment in Nodulocystic Acne with and without Polycystic Ovary Syndrome: Efficacy and Determinants of Relapse. Int $\mathrm{J}$ Dermatol. 2013;52(3):371-76

85. Çetinözman F, Yazgan AD, Elçin G, Yildiz BO. Insulin Sensitivity, Androgens and Isotretinoin Therapy in Women with Severe Acne. J Dermatolog Treat. 2014;25(2):119-22.

86. Vinaixa M, Rodriguez MA, Samino S, Díaz M, Beltran A, Mallol R, et al. Metabolomics Reveals Reduction of Metabolic Oxidation in Women with
Polycystic Ovary Syndrome after PioglitazoneFlutamide-Metformin Polytherapy. PLoS One. 2011;6(12):e29052. doi: 10.1371/journal.pone. 0029052 .

87. Du Q, Yang S, Wang YJ, Wu B, Zhao YY, Fan B. Effects of Thiazolidinediones on Polycystic Ovary Syndrome: A Meta-Analysis of Randomized Placebo-Controlled Trials. Adv Ther. 2012;29(9):763-74.

88. Jensterle Sever M, Kocjan T, Pfeifer M, Kravos NA, Janez A. Short-Term Combined Treatment with Liraglutide and Metformin Leads to Significant Weight Loss in Obese Women with Polycystic Ovary Syndrome and Previous Poor Response to Metformin. Eur J Endocrinol. 2014;170(3):451-59.

89. Thomson RL, Spedding S, Brinkworth GD, Noakes M, Buckley JD. Seasonal Effects on Vitamin D Status Influence Outcomes of Lifestyle Intervention in Overweight and Obese Women with Polycystic Ovary Syndrome. Fertil Steril. 2013;99(6):1779-85.

90. Celik O, Acbay O. Effects of Metformin plus Rosuvastatin on Hyperandrogenism in Polycystic Ovary Syndrome Patients with Hyperlipidemia and Impaired Glucose Tolerance. J Endocrinol Invest. 2012;35(10):905-10.

91. Macut D, Bjekić-Macut J, Savić-Radojević A. Dyslipidemia and Oxidative Stress in PCOS. Front Horm Res. 2013;40:51-63.

92. Lansdown A, Rees DA. The Sympathetic Nervous System in Polycystic Ovary Syndrome: A Novel Therapeutic Target? Clin Endocrinol (Oxf). 2012;77(6):791-801

93. Unfer V, Carlomagno G, Dante G, Facchinetti F. Effects of Myo-Inositol in Women with PCOS: A Systematic Review of Randomized Controlled Trials. Gynecol Endocrinol. 2012;28(7):509-15. 